\title{
Study of the $\mathrm{e}^{+} \mathrm{e}^{-}$to hadrons via ISR at BABAR*
}

\author{
E. P. Solodov ${ }^{1)}$ (for the BABAR collaboration) \\ Budker Institute of Nuclear Physics, SB RAS, 11 Lavrentieva ave., Novosibirsk, 630090, Russia
}

\begin{abstract}
Experimental data from the PEP-II B-factory at $10.6 \mathrm{GeV}$ center-of-mass (c.m.) energy, obtained via initial-state radiation (ISR) with the BABAR detector, are presented. The cross sections for many hadronic processes have been measured from the production threshold to $4-5 \mathrm{GeV}$ of the $\mathrm{e}^{+} \mathrm{e}^{-}$c.m. energy. The obtained data allow to study a number of intermediate states and determine the parameters of known resonances and their branching fractions. The exclusive cross section for some number of hadronic sub-processes are presented.
\end{abstract}

Key words ISR, $\mathrm{e}^{+} \mathrm{e}^{-}$, hadrons, BABAR

PACS 13.66.Bc, 14.40.-n, 13.75.-n

\section{Introduction}

The study of the $\mathrm{e}^{+} \mathrm{e}^{-} \rightarrow$ hadrons reaction at low c.m. energies using ISR technique became a powerful tool, thanks to the very high luminosity DAFNE, KEK-B and PEP-II colliders. Radiation of the highenergy photon from the initial particles allows a production of the hadronic system at energies much below the nominal machine energy $[1,2]$.

The BABAR [3] Collaboration has an intensive program to study the $\mathrm{e}^{+} \mathrm{e}^{-}$cross section at low c.m. energy using ISR, when the PEP-II operates at the $\Upsilon(4 S)$ resonance. A number of multi-hadron processes were studied and published. The measured cross sections made a major contribution to $R$ calculation during the last few years. Low energy hadron spectroscopy is another application of BABAR ISR study. It is shown, that spectroscopy data not only are absolutely necessary for the $R$ calculations (the total cross sections normally are the compositions of different states with different BF to certain modes), but give a lot of information about hadron dynamics in 1-2 GeV energy range. Multi-hadron events show many, sometimes unexpected structures which are not easy to separate - full partial wave analysis (PWA) is needed.

In this paper we present evidence of hadronic states in the $1.0-2.5 \mathrm{GeV}$ region, some of them are not listed in the PDG, as well as the already discov- ered $\mathrm{Y}(2175)$ in the $\phi(1020) \mathrm{f}_{0}(980)$ decay mode, confirmed by BES and Belle. The analysis is in progress, some new results should come out soon.

\section{Overview of the cross section mea- surements}

During the last few years the BABAR Collaboration published number of papers [4-13] on measurements of the total cross section for the reactions $\mathrm{e}^{+} \mathrm{e}^{-} \rightarrow 2 \pi, 3 \pi, 4 \pi, 5 \pi, 6 \pi, 2 \mathrm{~K} \pi, 2 \mathrm{~K} 2 \pi, 4 \mathrm{~K}, \mathrm{p} \overline{\mathrm{p}}, \Lambda \bar{\Lambda}$, $\mathrm{D} \overline{\mathrm{D}}, \ldots$ The overview of the measured cross sections is shown in Fig. 1. With the recent published data for the $\mathrm{e}^{+} \mathrm{e}^{-} \rightarrow 2 \pi$ process (see talk by Wen Feng) the BABAR data play dominant role in the muon $g-2 / 2$ calculation (see talk by M. Davier). The only channels are missing in this line are $\mathrm{e}^{+} \mathrm{e}^{-} \rightarrow \pi^{+} \pi^{-} 2 \pi^{0}$, for which only preliminary results were presented and $\mathrm{e}^{+} \mathrm{e}^{-} \rightarrow \mathrm{K}^{+} \mathrm{K}^{-}$reaction, which is still under study.

Studies of the exclusive channels of $\mathrm{e}^{+} \mathrm{e}^{-}$annihilation allow to determine such fundamental parameters as mass, width and leptonic width of various vector mesons. In addition to the low-lying resonances, such as the $\rho, \omega$ and $\phi$, where ISR studies can independently provide meaningful and still competitive information, they are indispensable for a much more precise than before investigation of the excited vector states. Moreover, detailed analysis of the dynamics shows that in many cases a multiparticle final state

\footnotetext{
Received 25 January 2010

* Supported by Russian Foundation of Basic Research (09-02-08413-3)

1) E-mail: solodov@inp.nsk.su

(C)2009 Chinese Physical Society and the Institute of High Energy Physics of the Chinese Academy of Sciences and the Institute of Modern Physics of the Chinese Academy of Sciences and IOP Publishing Ltd
} 


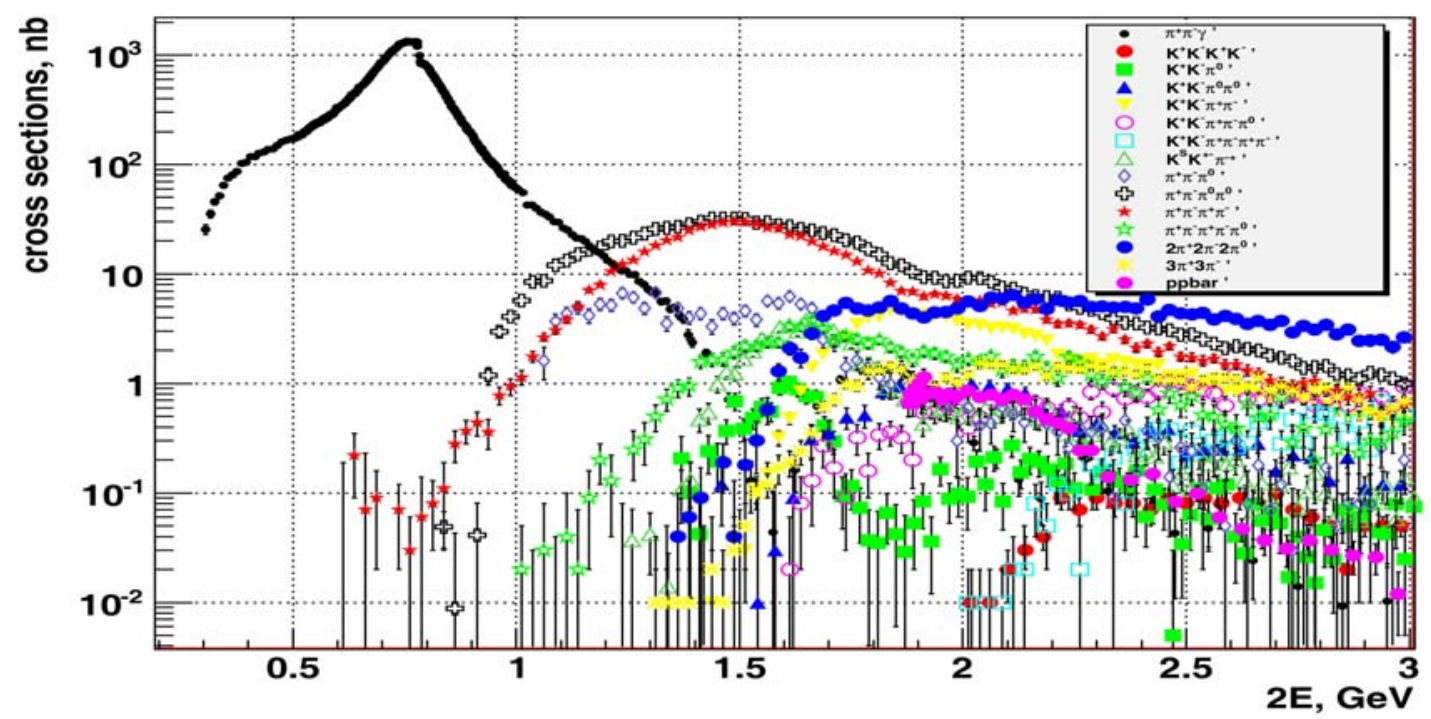

Fig. 1. Overview of the $\mathrm{e}^{+} \mathrm{e}^{-}$cross sections measured with the BABAR detector.

can be reached via different intermediate mechanisms.

For example, four pions can be produced via $\omega \pi^{0}$, $a_{1}(1260)^{ \pm} \pi^{\mp}, \rho^{0} f_{0}, \ldots$ and provide information on mesons with quantum numbers differing from those of vectors.

In the following sections we show a complexity of the internal substructures observed in some channels, which are used (where possible) to extract resonance parameters.

\section{Resonance physics}

\subsection{The $\mathrm{e}^{+} \mathrm{e}^{-} \rightarrow \pi^{+} \pi^{-}$reaction}

This reaction is dominated by production of the $\rho(770)$ resonance and the pion cross section behavior was relatively well studied up to about $1.4 \mathrm{GeV}$. The high statistic measurement [4] of the cross section up to $3.0 \mathrm{GeV}$ of c.m. energy is presented by BABAR for the first time. This energy range, shown in Fig. 2

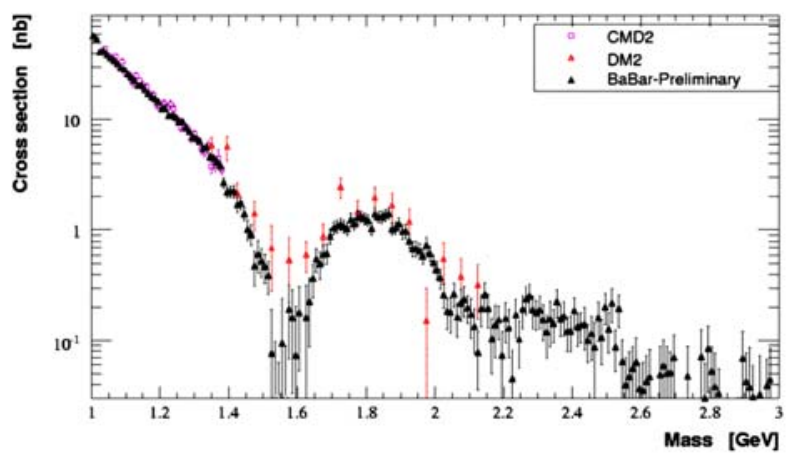

Fig. 2. The $\mathrm{e}^{+} \mathrm{e}^{-} \rightarrow \pi^{+} \pi^{-}$cross sections measured with the BABAR detector. demonstrates some statistically significant structures, not yet explained theoretically.

\subsection{The $\mathrm{e}^{+} \mathrm{e}^{-} \rightarrow \pi^{+} \pi^{-} \pi^{0}$ reaction}

The three pion reaction study in the ISR process was published by BABAR in Ref. [5] and measured $\mathrm{e}^{+} \mathrm{e}^{-} \rightarrow \pi^{+} \pi^{-} \pi^{0}$ cross section is dominated by the well known $\phi(1020), \omega(780)$ and $\mathrm{J} / \psi$ resonances. Large statistics allow the observation of two structures in the 1.0-2.0 GeV region in agreement with SND measurement [14] below $1.4 \mathrm{GeV}$ and in disagreement with old DM2 results [15] at higher energies.

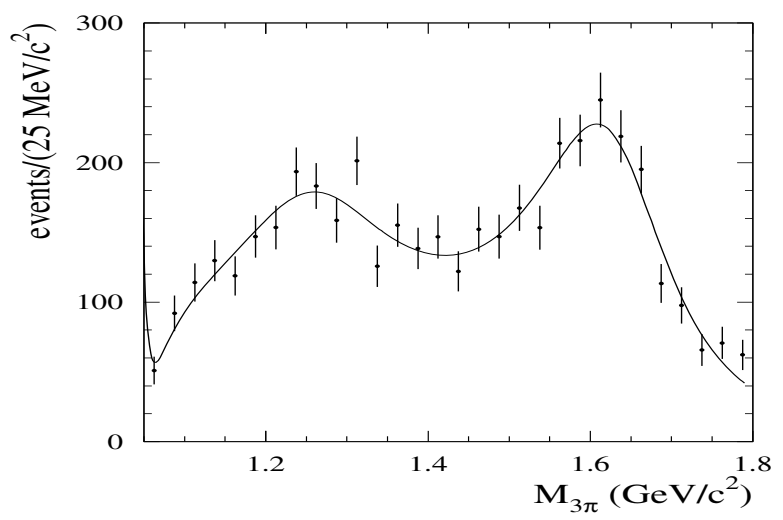

Fig. 3. The $\mathrm{m}\left(\pi^{+} \pi^{-} \pi^{0}\right)$ distribution for the $\mathrm{e}^{+} \mathrm{e}^{-} \rightarrow \pi^{+} \pi^{-} \pi^{0}$ reaction measured with the BABAR detector. Two-resonance fit is shown.

We fit this region (see Fig. 3) assuming the presence of two excited $\omega(1420)$ and $\omega(1650)$ [21] states and determine their parameters. Note, that the parameters of these states are still not well determined 
and in our case they strongly depend on relative phases with $\omega(780)$ and $\phi(1020)$ resonances having much larger decay rate to this mode.

\subsection{The $\mathrm{e}^{+} \mathrm{e}^{-} \rightarrow \pi^{+} \pi^{-} \pi^{+} \pi^{-}, \pi^{+} \pi^{-} 2 \pi^{0}$ reac- tions}

These reactions have the largest cross sections in the studied energy region after $\mathrm{e}^{+} \mathrm{e}^{-} \rightarrow \pi^{+} \pi^{-}$. They were studied with the BABAR detector $[6,7]$ in much wider energy range than any previous experiment. The intermediate resonance production of $\omega \pi^{0}$ in the $\pi^{+} \pi^{-} 2 \pi^{0}$ final state and $a_{1} \pi$ dominance in both reactions were previously reported. The cross section shape for both reactions shows a wide structure peaked at $1.5 \mathrm{GeV}$ which cannot be described as a single resonance and a "shoulder" just below $2 \mathrm{GeV}$ which also has no explanation.

The large number of events collected in the BABAR experiment allows to demonstrate an evidence of some number of other intermediate states such as $\rho(770) \mathrm{f}_{0}(980), \rho(770) \mathrm{f}_{0}(1270)$ and surpris- ingly large $\rho^{+} \rho^{-}$(see Fig. 4). Simultaneous study of charged and neutral modes gives an unique possibility for the partial wave analysis (PWA), not done yet for these reactions.

\subsection{The $\mathbf{e}^{+} \mathbf{e}^{-} \rightarrow \mathbf{K}^{+} \mathbf{K}^{-} \pi^{0}, \mathbf{K}_{\mathrm{S}} \mathbf{K} \pi, \mathbf{K}^{+} \mathbf{K}^{-} \boldsymbol{\eta}$ re- actions}

The BABAR Collaboration performs a combined PWA of these reactions [10] which allows to separate iso-scalar and iso-vector components of the cross sections for the quasi-two-body dominant $\mathrm{K}^{*}(892) \mathrm{K}$ and $\mathrm{K}^{*}(1430) \mathrm{K}$ intermediate states. The iso-scalar component is dominated by the $\mathrm{e}^{+} \mathrm{e}^{-} \rightarrow \phi(1680)$ resonance production. Again, large statistic allows to extract relatively small cross sections of $\mathrm{e}^{+} \mathrm{e}^{-} \rightarrow$ $\phi(1020) \eta$ and $\mathrm{e}^{+} \mathrm{e}^{-} \rightarrow \phi(1020) \pi^{0}$ reactions (see Fig. 5) which were not previously studied. These reactions are very good to search for the exotic states and BABAR demonstrates a hint of a possible state at about $1.5 \mathrm{GeV}$ in the OZI suppressed $\phi(1020) \pi^{0}$ channel.
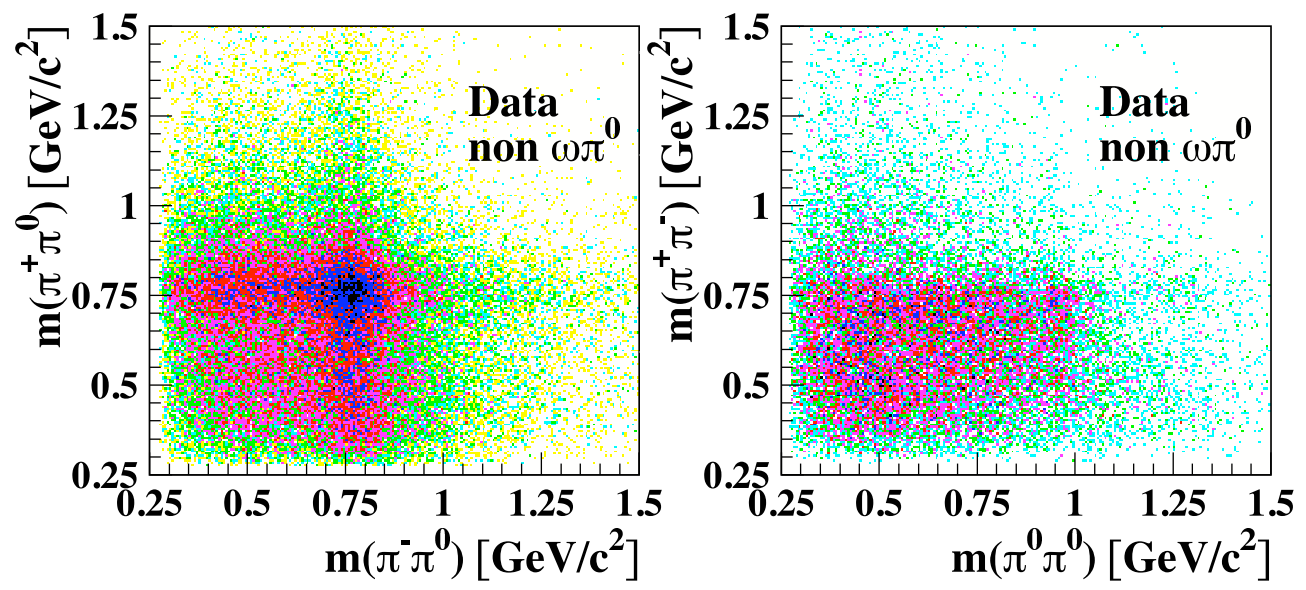

Fig. 4. Scatter plots $\mathrm{m}\left(\pi^{-} \pi^{0}\right)$ vs. $\mathrm{m}\left(\pi^{-} \pi^{0}\right)$ (left) and $\mathrm{m}\left(\pi^{+} \pi^{-}\right)$vs. $\mathrm{m}\left(\pi^{0} \pi^{0}\right)$ (right) for the $\mathrm{e}^{+} \mathrm{e}^{-} \rightarrow \pi^{+} \pi^{-} \pi^{0} \pi^{0}$ reaction measured with the BABAR detector.
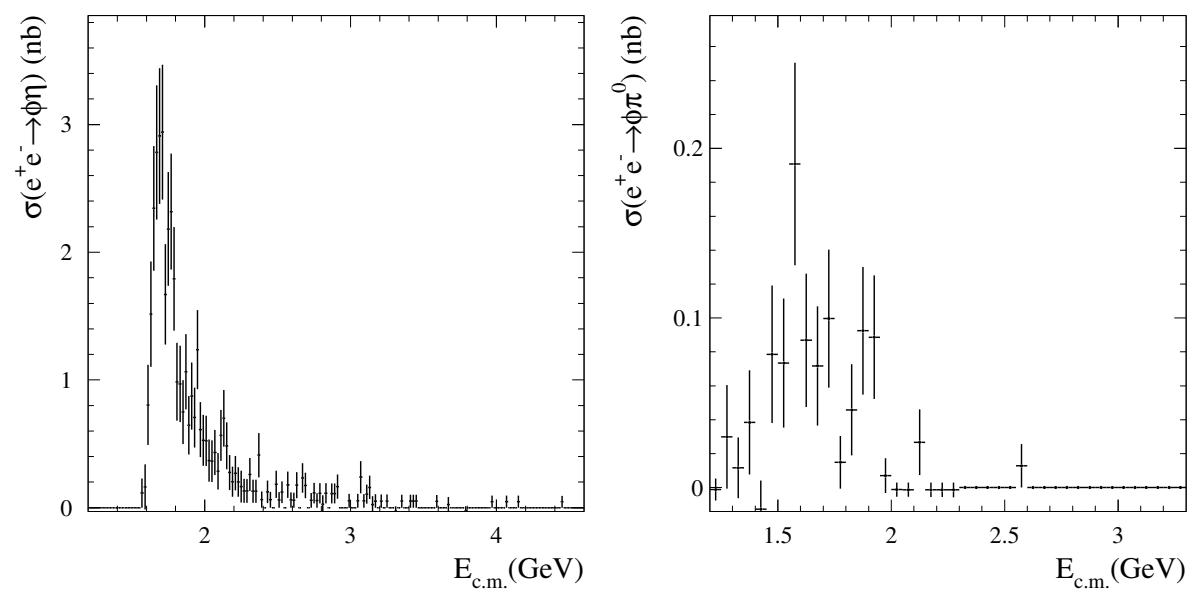

Fig. 5. The $\mathrm{e}^{+} \mathrm{e}^{-} \rightarrow \phi \eta, \phi \pi^{0}$ cross sections measured with the BABAR detector. 


\subsection{The $\mathrm{e}^{+} \mathrm{e}^{-} \rightarrow \mathrm{K}^{+} \mathrm{K}^{-} \pi^{+} \pi^{-}\left(\pi^{0} \pi^{0}\right)$ reactions}

The only measurement available refer to the study on the fully charged mode, performed by DM2 with about 100 times less data. The $\mathrm{e}^{+} \mathrm{e}^{-} \rightarrow$ $\mathrm{K}^{+} \mathrm{K}^{-} \pi^{0} \pi^{0}$ reaction never was studied before. It is shown by BABAR that these reactions are dominated by $\mathrm{K}^{*}(892)^{0} \mathrm{~K}^{ \pm} \pi^{\mp}$ and $\mathrm{K}^{*}(892)^{\mp} \mathrm{K}^{ \pm} \pi^{0}$ intermediate states [11] (see Fig. 6), but $\mathrm{K}^{*}(892) \overline{\mathrm{K}}^{*}(892)$ decay mode is not seen.

For the charged mode the contributions from $\mathrm{K}_{2}^{*}(1430) \mathrm{K} \pi$ and from $\mathrm{K}_{1}(1230)^{ \pm} \mathrm{K}^{\mp}, \mathrm{K}_{1}(1400)^{ \pm} \mathrm{K}^{\mp}$ decaying to $K^{ \pm} \rho(770)$ are seen. The full PWA is needed to disintegrate the measured inclusive cross section to observed modes.

The BABAR data allow to study relatively small contribution of $\phi(1020) \pi^{+} \pi^{-}\left(\pi^{0} \pi^{0}\right)$ intermediate states. Fig. 7 (left) shows $\mathrm{m}\left(\pi^{+} \pi^{-}\right)$distribution for these events, which can be fitted with a superposition of two scalar $\mathrm{f}_{0}(980)$ and $\mathrm{f}_{0}(600)$ resonances. By selecting events associated with $\mathrm{f}_{0}(980)$ peak, the cross section for the $\mathrm{e}^{+} \mathrm{e}^{-} \rightarrow \phi \mathrm{f}_{0}$ reaction is obtained (Fig. 7(right)) with a structure at about $2.175 \mathrm{GeV}$. It is interpreted as a resonance with about $80 \mathrm{MeV}$ width, later confirmed by BES and Belle experiments. The nature of this relatively narrow $\mathrm{Y}(2175)$ [22] state is not clear.

\subsection{The $\mathrm{e}^{+} \mathrm{e}^{-} \rightarrow 5$ hadrons reactions}

The BABAR detector studied $2\left(\pi^{+} \pi^{-}\right) \pi^{0}$, $2\left(\pi^{+} \pi^{-}\right) \eta, \mathrm{K}^{+} \mathrm{K}^{-} \pi^{+} \pi^{-} \pi^{0}$ and $\mathrm{K}^{+} \mathrm{K}^{-} \pi^{+} \pi^{-} \eta$ reactions produced via ISR [8].
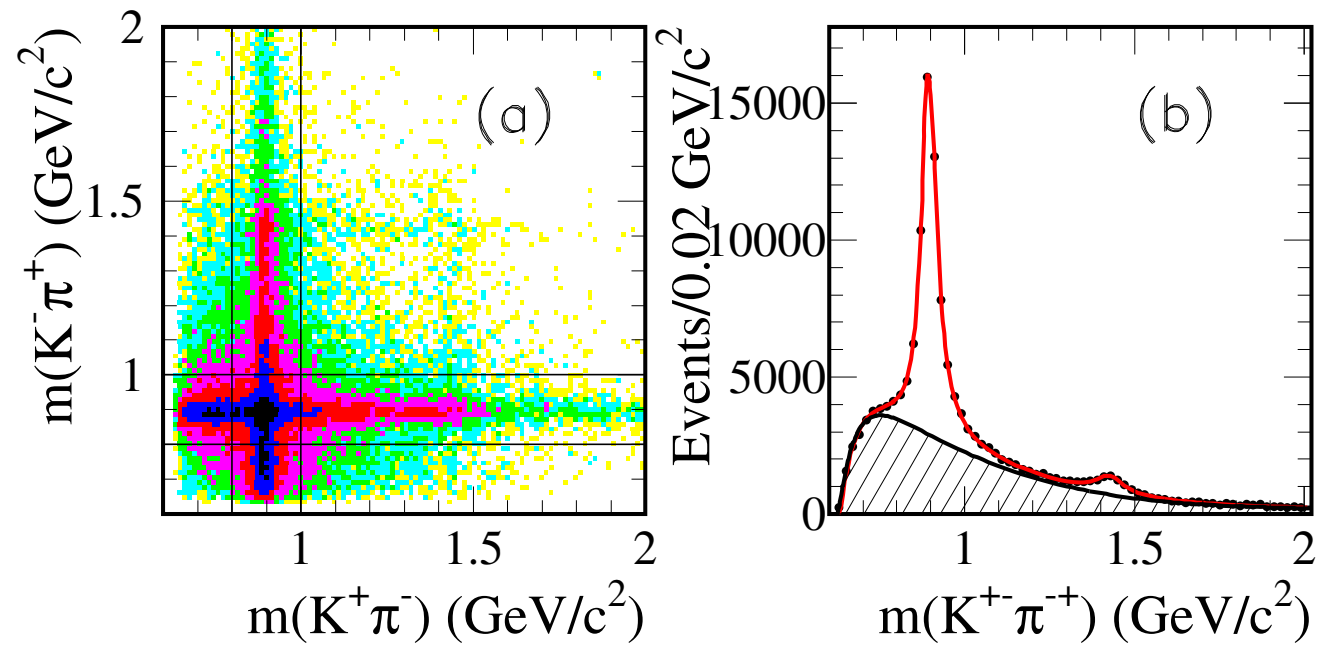

Fig. 6. Scatter plots $\mathrm{m}\left(\mathrm{K}^{-} \pi^{+}\right)$vs. $\mathrm{m}\left(\mathrm{K}^{+} \pi^{-}\right)$(a) and projection $\mathrm{m}\left(\mathrm{K}^{ \pm} \pi^{\mp}\right)$ plot (b, two entries per event) for the $\mathrm{e}^{+} \mathrm{e}^{-} \rightarrow \mathrm{K}^{+} \mathrm{K}^{-} \pi^{+} \pi^{-}$reaction.
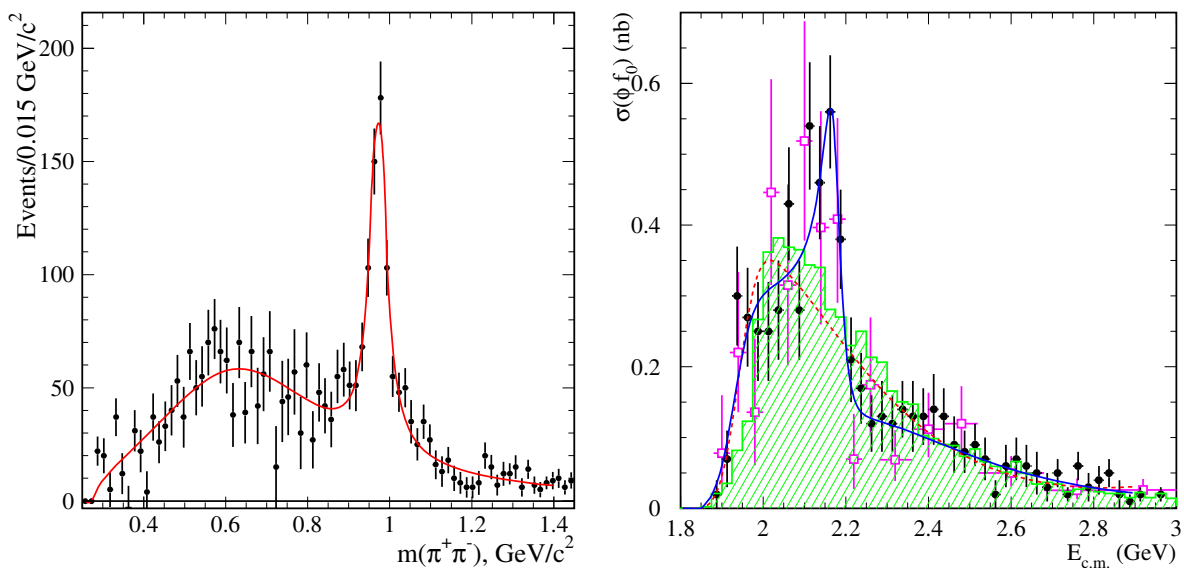

Fig. 7. Left: The $m\left(\pi^{+} \pi^{-}\right)$distribution for the $\mathrm{e}^{+} \mathrm{e}^{-} \rightarrow \phi(1020) \pi^{+} \pi^{-}$reaction. Right: The $\mathrm{e}^{+} \mathrm{e}^{-} \rightarrow \phi \mathrm{f}_{0}$ cross sections measured with the BABAR detector. 
The $\mathrm{e}^{+} \mathrm{e}^{-} \rightarrow 2\left(\pi^{+} \pi^{-}\right) \pi^{0}$ reaction has the largest cross section and has very distinctive $\omega \pi^{+} \pi^{-}$and $\eta \pi^{+} \pi^{-}$intermediate states seen in $\mathrm{m}\left(\pi^{+} \pi^{-} \pi^{0}\right)$ distribution of Fig. 8(left). They were studied before, but BABAR experiment [8] performed the most accurate measurement of the cross sections for these two channels. The two pions from the $\eta \pi^{+} \pi^{-}$reaction predominantly form $\rho(770)$ while for the $\omega \pi^{+} \pi^{-}$ reaction the $\mathrm{f}_{0}(980)$ signal is seen in two-pion invariant mass (see Fig. 8(right)) and, for the first time, the contribution of $\omega \mathrm{f}_{0}(980)$ was extracted and the cross section was measured (shown in Fig. 9(left)). The fit of the $\omega \pi^{+} \pi^{-}$cross section after subtraction of the $\omega \mathrm{f}_{0}(980)$ contribution can be used for more accurate information about $\omega(1400)$ and $\omega(1650)$ (see Fig. 10). The obtained parameters are in good agreement with those obtained from the $\pi^{+} \pi^{-} \pi^{0}$ channel and confirm an $\omega(1650)$ width more narrow than what is listed in the previous edition of the PDG tables.
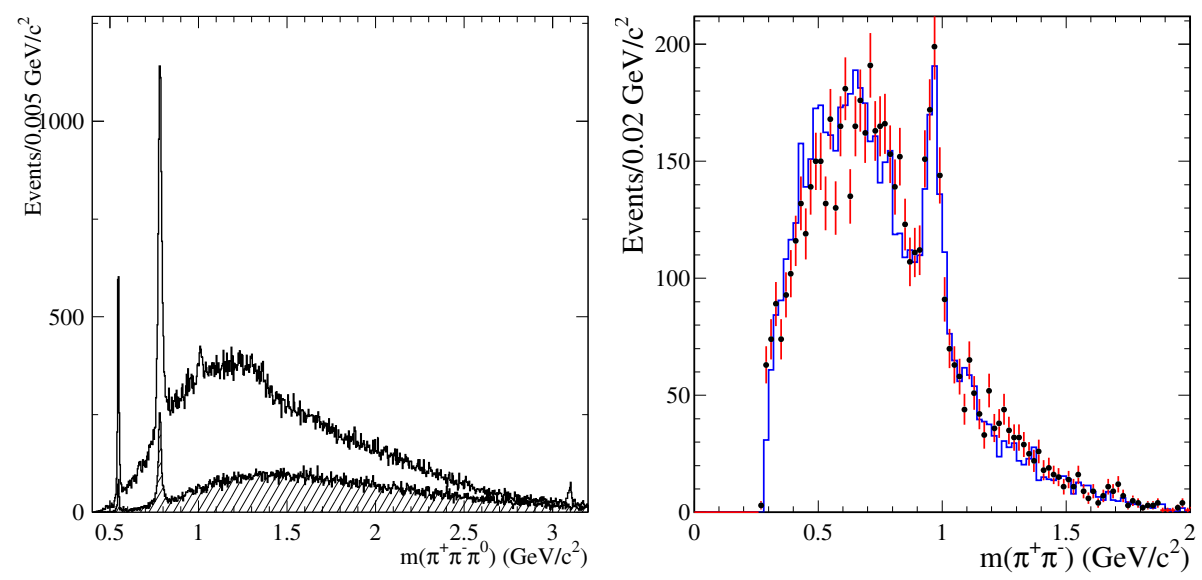

Fig. 8. Left: The $m\left(\pi^{+} \pi^{-} \pi^{0}\right)$ distribution for the $2\left(\pi^{+} \pi^{-}\right) \pi^{0}$ events. Right: The $m\left(\pi^{+} \pi^{-}\right)$distribution for all $\omega \pi^{+} \pi^{-}$events.
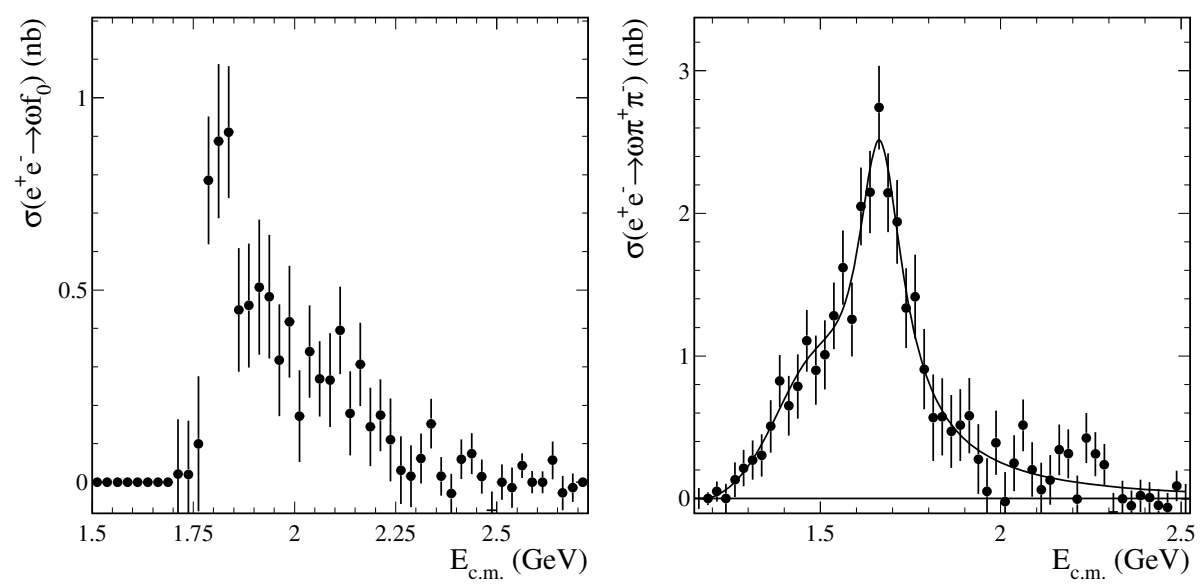

Fig. 9. Left: The $\mathrm{e}^{+} \mathrm{e}^{-} \rightarrow \omega f_{0}(980)$ cross section. Right: The two Breit-Wigner fit of $\omega \pi^{+} \pi^{-}$cross section with $\omega f_{0}(980)$ contribution subtracted.

The $\omega \pi^{+} \pi^{-}$and $\eta \pi^{+} \pi^{-}$do not explain the total $\mathrm{e}^{+} \mathrm{e}^{-} \rightarrow 2\left(\pi^{+} \pi^{-}\right) \pi^{-}$cross section as shown in Fig. 10(left). When $\omega \pi^{+} \pi^{-}$and $\eta \pi^{+} \pi^{-}$contributions are excluded, the $m\left(\pi^{+} \pi^{-}\right)$and $m\left(\pi^{ \pm} \pi^{0}\right)$ distributions (see Fig. 10(right)) demonstrate equal number of charged and neutral $\rho(770)$ per event with the remaining three-pion mass around $1240 \mathrm{MeV} / c^{2}$ and about $400 \mathrm{MeV}$ width. It is shown, that $\mathrm{e}^{+} \mathrm{e}^{-} \rightarrow \rho 3 \pi$ cross section is dominated by $\rho(770) \mathrm{X}(1240)$ production with $\mathrm{X}(1240) \rightarrow \rho(770) \pi$ decay. The best candidates for $\mathrm{X}(1240)$ are $\pi(1300)$ and $\mathrm{a}_{1}(1260)$ [21]. The detailed PWA is needed for more definite statement. The $\mathrm{e}^{+} \mathrm{e}^{-} \rightarrow 2\left(\pi^{+} \pi^{-}\right) \eta$ was studied with the BABAR detector for the first time. Fig. 11(left) shows $m\left(\eta \pi^{+} \pi^{-}\right)$distribution for these events, demonstrating contribution from $\eta(958) \pi^{+} \pi^{-}$ 
reaction. Narrow $\eta(958)$ signal allows to extract $\mathrm{e}^{+} \mathrm{e}^{-} \rightarrow \eta(958) \pi^{+} \pi^{-}$cross section (Fig. 11(right)) (dominated by $\eta(958) \rho(770))$. The cross section has a resonance structure and can be interpreted as $\rho(2150)$ production. Another definite structure seen in Fig. 11(left) and shown in Fig. 12(left) was inter-

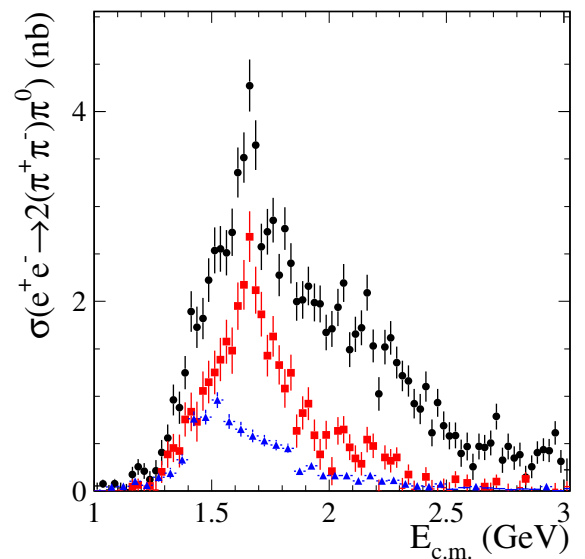

preted as production of $\mathrm{f}_{1}(1285) \pi^{+} \pi^{-}$intermediate state. After non-resonant background subtraction, the calculated $\mathrm{e}^{+} \mathrm{e}^{-} \rightarrow \mathrm{f}_{1}(1285) \pi^{+} \pi^{-}$cross section is shown in Fig. 12(right). A resonance structure also can be interpreted as $\rho(2150)$ production.

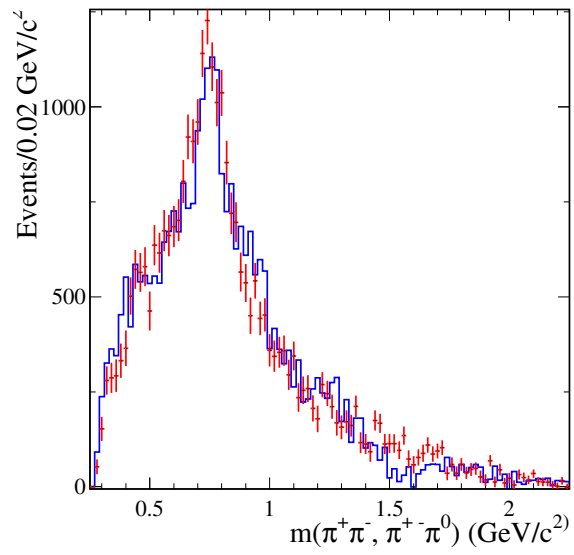

Fig. 10. Left: The $\mathrm{e}^{+} \mathrm{e}^{-} \rightarrow 2\left(\pi^{+} \pi^{-}\right) \pi^{0}$ cross section and contribution from $\omega \pi^{+} \pi^{-}$(squares) and $\eta \pi^{+} \pi^{-}$ (triangles) reactions. Right: The $m\left(\pi^{+} \pi^{-}\right)$(points) and $m\left(\pi^{ \pm} \pi^{0}\right)$ (histogram) distributions for $2\left(\pi^{+} \pi^{-}\right) \pi^{0}$ events with $\omega \pi^{+} \pi^{-}$and $\eta \pi^{+} \pi^{-}$contributions excluded.
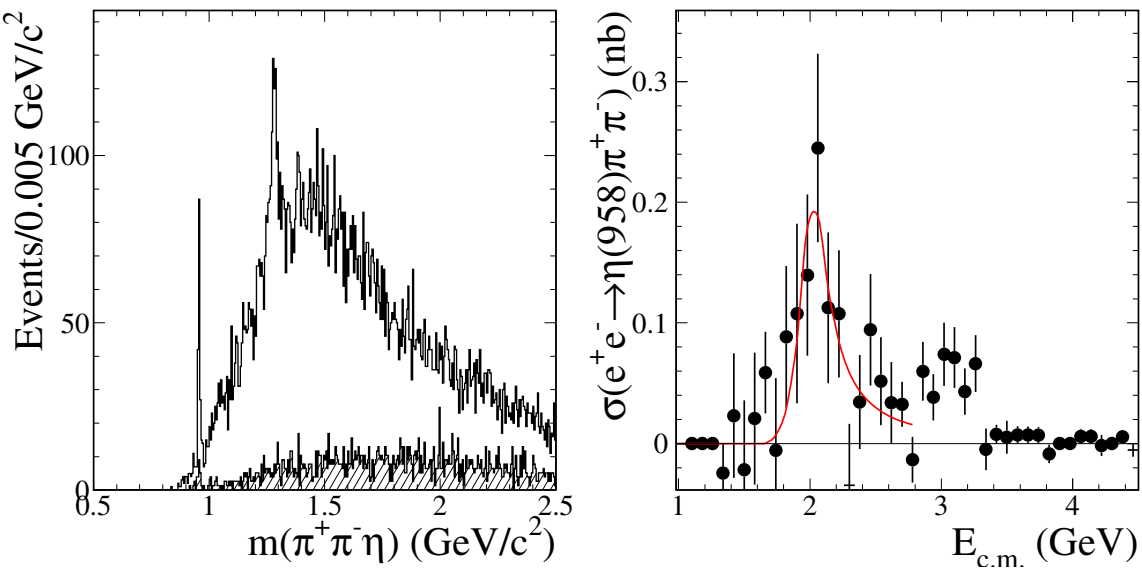

Fig. 11. Left: The $m\left(\eta \pi^{+} \pi^{-}\right)$distribution for the $2\left(\pi^{+} \pi^{-}\right) \eta$ events. Right: The $\mathrm{e}^{+} \mathrm{e}^{-} \rightarrow \eta(958) \pi^{+} \pi^{-}$cross section and Breit-Wigner fit.
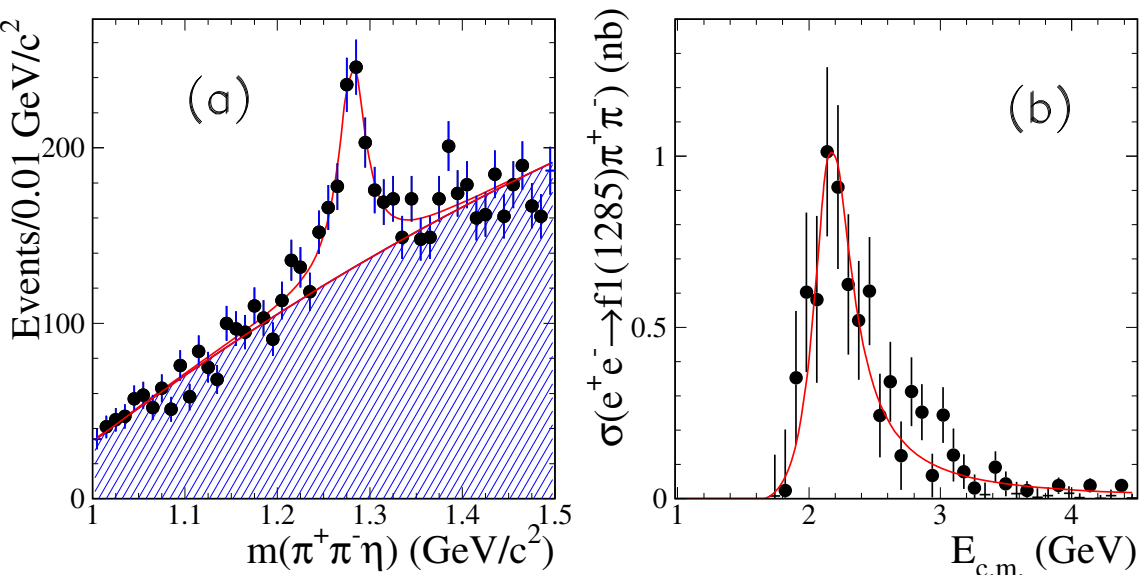

Fig. 12. The $m\left(\eta \pi^{+} \pi^{-}\right)$distribution (a) for the $2\left(\pi^{+} \pi^{-}\right) \eta$ events. The $\mathrm{e}^{+} \mathrm{e}^{-} \rightarrow \mathrm{f}_{1}(1285) \pi^{+} \pi^{-}$cross section (b) and Breit-Wigner fit. 
The cross sections for the $\mathrm{e}^{+} \mathrm{e}^{-} \rightarrow \mathrm{K}^{+} \mathrm{K}^{-} \pi^{+} \pi^{-} \pi^{0}$ reaction was measured by $\mathrm{BABAR}$ for the first time. The three-pion and two-kaon invariant masses are shown in Fig. 13. The signals from $\eta$ and $\omega$ are well seen (small signal from $\phi$ ) in three-pion mass distribution with strong $\phi$ signal in $m\left(\mathrm{~K}^{+} \mathrm{K}^{-}\right)$distribution. Fig. 14 shows the calculated cross sections for the $\mathrm{e}^{+} \mathrm{e}^{-} \rightarrow \phi \eta($ left $)$ and $\mathrm{e}^{+} \mathrm{e}^{-} \rightarrow \omega \mathrm{K}^{+} \mathrm{K}^{-}$(right) subprocesses. The first one is in good agreement with

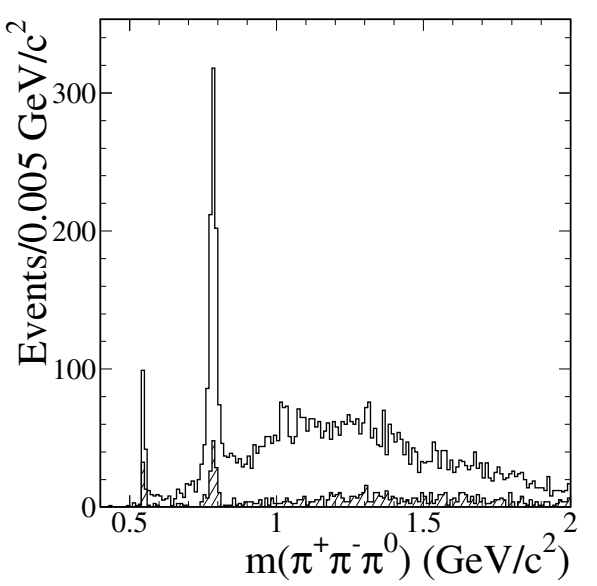

obtained in the $\eta \rightarrow \gamma \gamma$ mode [10]. It is first measurement for the second process with possible structures, but the only significant one is $\mathrm{J} / \psi \rightarrow \omega \mathrm{K}^{+} \mathrm{K}^{-}$signal.

The cross sections for the $\mathrm{e}^{+} \mathrm{e}^{-} \rightarrow \mathrm{K}^{+} \mathrm{K}^{-} \pi^{+} \pi^{-} \eta$ reaction was also measured by BABAR for the first time. The cross section is small and the only significant signal observed was $\mathrm{e}^{+} \mathrm{e}^{-} \rightarrow \phi \eta(958)$ in the studied $\mathrm{K}^{+} \mathrm{K}^{-} \pi^{+} \pi^{-} \eta$ final state.

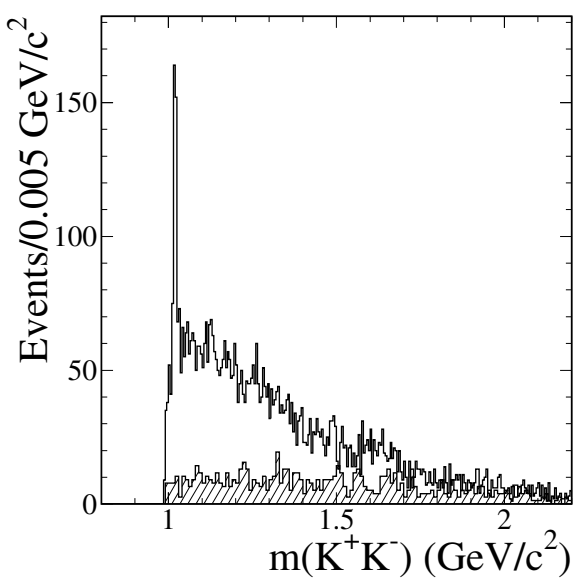

Fig. 13. The $m\left(\pi^{+} \pi^{-} \pi^{0}\right)$ (left) and $m\left(\mathrm{~K}^{+} \mathrm{K}^{-}\right)$(right) distributions for the $\mathrm{K}^{+} \mathrm{K}^{-} \pi^{+} \pi^{-} \pi^{0}$ events.
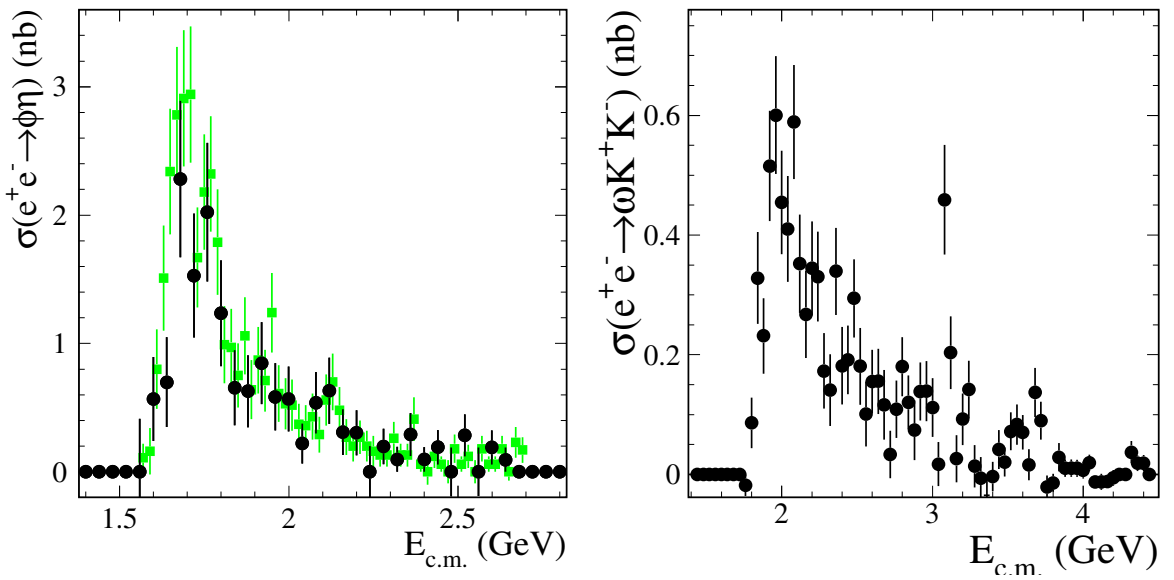

Fig. 14. The $\mathrm{e}^{+} \mathrm{e}^{-} \rightarrow \phi \eta$ (left) and $\mathrm{e}^{+} \mathrm{e}^{-} \rightarrow \omega \mathrm{K}^{+} \mathrm{K}^{-}$(right) cross sections for the $\mathrm{K}^{+} \mathrm{K}^{-} \pi^{+} \pi^{-} \pi^{0}$ events.

\subsection{The $\mathrm{e}^{+} \mathrm{e}^{-} \rightarrow 3\left(\pi^{+} \pi^{-}\right), 2\left(\pi^{+} \pi^{-} \pi^{0}\right)$ reac- tions}

These reactions were studied before by several experiments but with almost 100 times smaller statistic and very large systematic uncertainties. The BABAR detector performed measurement of the six-pion $\mathrm{e}^{+} \mathrm{e}^{-}$ production [9] from the threshold to about $5 \mathrm{GeV}$ using ISR events. The systematical uncertainty was dramatically reduced. One of the interesting obser- vation in the $3\left(\pi^{+} \pi^{-}\right)$was a presence of only one $\rho(770)^{0}$ per event in many possible $\pi^{+} \pi^{-}$combinations. No other intermediate resonance signals were observed. For the $2\left(\pi^{+} \pi^{-} \pi^{0}\right)$ final state we also observe only one $\rho(770)^{0}$ or $\rho(770)^{ \pm}$per event with expected $1 / 2$ ratio.

In the $2\left(\pi^{+} \pi^{-} \pi^{0}\right)$ final state we observe the $\eta$ and $\omega$ signals in the $\pi^{+} \pi^{-} \pi^{0}$ mass distribution. Also a small fraction of events corresponds to a correlated production of $\eta$ and $\omega$. The $\mathrm{e}^{+} \mathrm{e}^{-} \rightarrow \omega \eta$ cross section 
was measured for the first time (Fig. 15(left)) and the resonance structure fitted with the Breit-Wigner function is assumed to be from $\omega(1650) \rightarrow \omega(782) \eta$ reaction. The $\mathrm{e}^{+} \mathrm{e}^{-} \rightarrow 2\left(\pi^{+} \pi^{-} \pi^{0}\right)$ and $\mathrm{e}^{+} \mathrm{e}^{-} \rightarrow$ $3\left(\pi^{+} \pi^{-}\right)$cross sections have very similar energy behavior (see Fig. 16) and the ratio of these two cross section is close to uniform with $3.98 \pm 0.06 \pm 0.41$ value. We also observe a dip structure just blow $2 \mathrm{GeV}$ in total six-pion cross sections which was first ob- served by FENICE [23] experiment and confirmed by FOCUS [24].

We fit this structure with the Breit-Wigner function fully coherent with smooth non-resonant background shown in Fig. 16. The structre has a mass of about $1.88 \mathrm{GeV} / c^{2}$ and a width of about $0.1 \mathrm{GeV}$ (wider than that from FOCUS) and could be interpreted as a proton-anti-proton ground state.
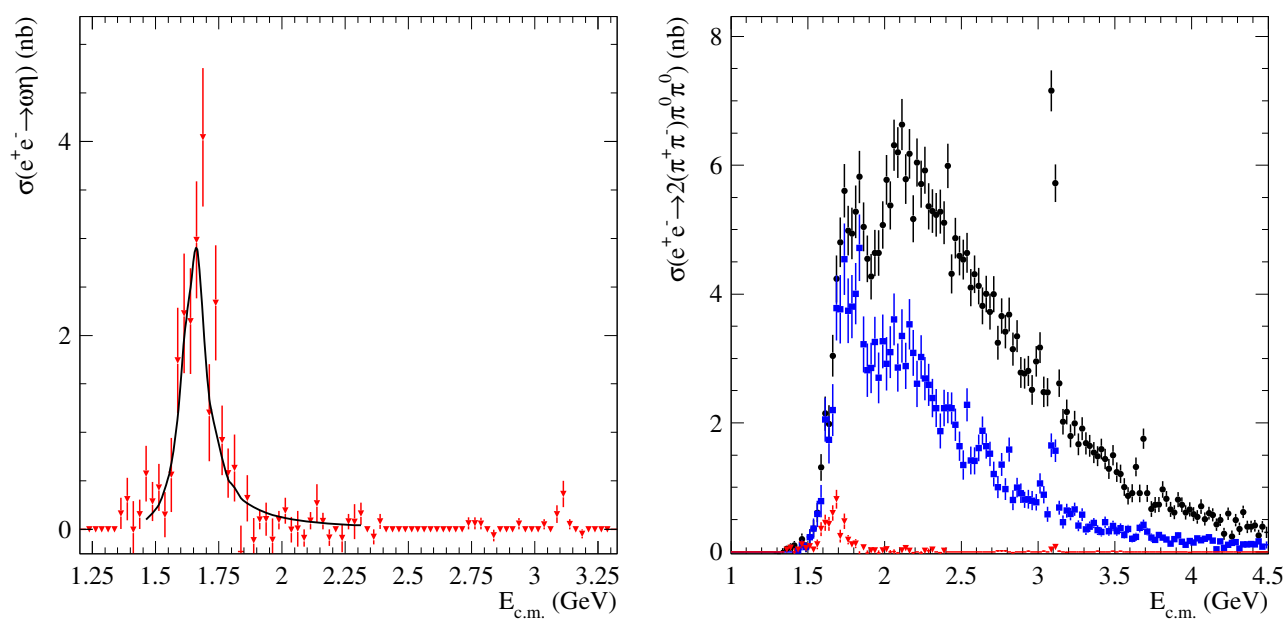

Fig. 15. The $\mathrm{e}^{+} \mathrm{e}^{-} \rightarrow \omega \eta$ cross section (left) and contribution of $\mathrm{e}^{+} \mathrm{e}^{-} \rightarrow \omega \pi^{+} \pi^{-} \pi^{0}$ and $\mathrm{e}^{+} \mathrm{e}^{-} \rightarrow \omega \eta$ cross sections to all $\mathrm{e}^{+} \mathrm{e}^{-} \rightarrow 2\left(\pi^{+} \pi^{-} \pi^{0}\right)$ events (right).
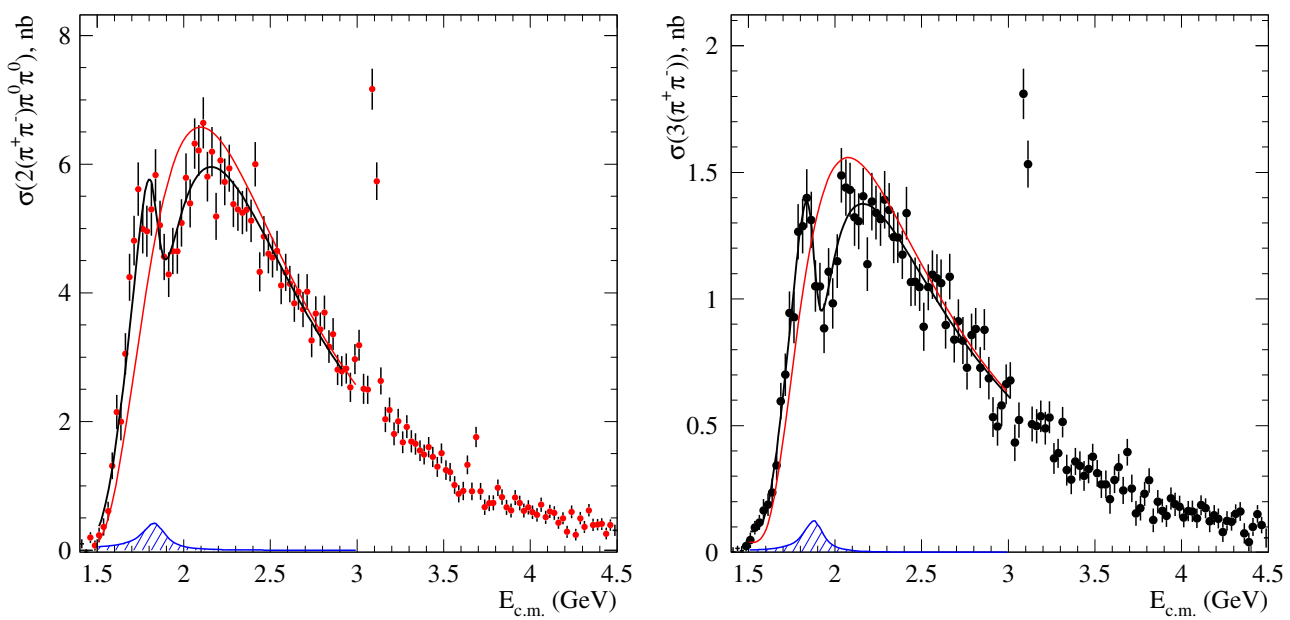

Fig. 16. The $\mathrm{e}^{+} \mathrm{e}^{-} \rightarrow 2\left(\pi^{+} \pi^{-} \pi^{0}\right)$ cross section (left) and the $\mathrm{e}^{+} \mathrm{e}^{-} \rightarrow 3\left(\pi^{+} \pi^{-}\right)$cross section (right) with fit to (anti-)resonance function. A resonance responsible for the "dip" is shown shaded.

\section{Summary}

The BABAR detector has an intensive program for study low energy $\mathrm{e}^{+} \mathrm{e}^{-}$cross sections using ISR events. Numerous $\mathrm{e}^{+} \mathrm{e}^{-} \rightarrow$ hadrons cross sections have been measured with accuracy better than all previous direct $\mathrm{e}^{+} \mathrm{e}^{-}$experiments. The large integrated luminosity provided by PEPII collider and the excellent BABAR detector performance allow to study many multi-hadron final 
states and extract intermediate resonance structures which includes mesons $(\pi, \mathrm{K}, \mathrm{D})$ and barions $(\mathrm{p}, \Lambda, \Sigma)$. The parameters of many known states $(\omega(1400), \omega(1650), \phi(1680) \ldots)$ and new resonance candidates $(\rho(2150), \mathrm{X}(1880) \ldots)$ were measured with accuracy better than current table val- ues. New resonances have been discovered via ISR: $\mathrm{Y}(4260), \mathrm{Y}(4320), \mathrm{Y}(2175)$. But in many cases the internal structures are very complicated and sophisticated PWA is needed to extract coupling constants and parameters of seen resonances. It is a challenge for future studies.

\section{References}

1 Benayoun M et al. Mod. Phys. Lett. A, 1999, 14: 2605

2 Bonneau G, Martin F. Nucl. Phys. B, 1971, 27: 381

3 BABAR collaboration, Aubert B et al. Nucl. Instr. and Meth. A, 2002, 479: 1

4 BABAR collaboration, Aubert B et al. Phys. Rev. Lett., 2009, 103: 231801

5 BABAR collaboration, Aubert B et al. Phys. Rev. D, 2004, 70: 072004

6 BABAR collaboration, Aubert B et al. Phys. Rev. D, 2005, 71: 052001

7 Druzhinin V P. arxiv: hep-ex/0710.3455

8 BABAR collaboration, Aubert B et al. Phys. Rev. D, 2007, 76: 092005; 2008, 77: 119902

9 BABAR collaboration, Aubert B et al. Phys. Rev. D, 2006, 73: 052003

10 BABAR collaboration, Aubert B et al. Phys. Rev. D, 2008, 77: 092002

11 BABAR collaboration, Aubert B et al. Phys. Rev. D, 2006, 74: 091103

12 BABAR collaboration, Aubert B et al. Phys. Rev. D, 2009, 79: 092001
13 BABAR collaboration, Aubert B et al. Phys. Rev. D, 2006, 73: 012005 (2006); hep-ex/05112023

14 SND collaboration, Achasov M N et al. Phys. Rev. D, 2002, 66: 032001

15 DM2 collaboration, Antonelli A et al. Z. Phys. C, 1992, 56: 15

16 Chernyak V L, Zhitnitsky A R, JETP Lett., 1977, 25: 510

17 PS170 collaboration, Bardin G et al. Nucl. Phys. B, 1994, 411: 3

18 BABAR collaboration, Aubert B et al. Phys. Rev. D, 2007, 76: 092006; hep-ex/0709.1988.

19 DM2 collaboration, Bisello D et al. Z. Phys. C, 1990, 48: 23

20 Chernyak V L et al. Z. Phys. C, 1989, 42: 569

21 Amsler C et al (Particle Data Group). Phys. Lett. B, 2008, 667: 1; 2009 partial update for the 2010 edition

22 Gomez-Avila S, Napsuciale M, Oset E. arXiv:0711.4147 [hep-ph]; Napsuciale M et al. Phys. Rev. D, 2007, 76: 074012, e-Print: arXiv:0706.2972 [hep-ph]

23 Clegg A B, Donnachie A. Z. Phys. C, 1990, 45: 677

24 FOCUS collaboration, Frabetti P L et al. Phys. Lett. B, 2000, 514: 240 